\title{
Thanks to reviewers in 2014
}

Published online: 17 October 2014

(C) Springer-Verlag Berlin Heidelberg 2014

The mission of Analytical and Bioanalytical Chemistry (ABC) is the communication of excellent research work from all fields of analytical and bioanalytical chemistry. Clearly, a scientific journal's greatest responsibility toward the scientific community is to ensure that all contributions accepted for publication are rigorously but fairly reviewed. For this reason, all manuscripts published in the journal are reviewed by expert referees. The final quality of papers accepted for publication depends to a considerable extent on the reviewers, who give valuable constructive criticism to the authors. The input and dedication of all our reviewers are highly appreciated by both the editors and the authors.

As a small token of our thanks, those reviewers who have been most supportive in assessing manuscripts for Analytical and Bioanalytical Chemistry during 2014 will be offered a Springer book of their choice up to the value of 150 euros.

At the end of 2014, we gratefully acknowledge the valuable support of the following scientists who have reviewed papers for the journal during the past 12 months:

Aamouche, A, Safi

Aboul-Enein, HY, Cairo

Absalon, C, Talence

Abu-Reidah, IM, Til

Acree, WE Jr., Denton, TX

Adam, V, Brno

Adami, G, Trieste

Adams, AA, Washington, DC

Adams, FC, Wilrijk

Adams, KM, Rockville, MD

Aga, DS, Buffalo, NY

Aguilar-Caballos, MdlP, Córdoba

Ahting, M, Dessau-Rosslau

Akgöz, M, Kocaeli

Albert, K, Tübingen

Alberts, D, Oviedo

Alcalà, M, Bellaterra

Alder, L, Berlin

Aldini, G, Milan

Alexandrov, T, Bremen

Algar, WR, Mississauga, ON

Almirall, JR, Miami, FL

Altmann, F, Vienna

Alvarez, D, Columbia, MO

Alves, A, Porto

Amann, A, Innsbruck

Ambach, L, Bern
Amjadi, M, Tabriz

Amoresano, A, Naples

Andersen, JET, Kongens Lyngby

Anderson, JL, Toledo, $\mathrm{OH}$

Andersson, JT, Münster

Andrade Garda, JM, Corunna

Andres-Lacueva, C, Barcelona

Andrisano, V, Bologna

Anfossi, L, Turin

Anoardo, E, Córdoba

Aranda, M, Concepcion

Arce Osuna, M, El Marques

Arduini, F, Rome

Ariese, F, Amsterdam, NH

Arisi, ACM, Florianópolis

Arlinghaus, HF, Münster

Armstrong, DW, Arlington, TX

Aroca, RF, Windsor, ON

Arriaga, EA, Minneapolis, MN

Arrigan, DWM, Perth, WA

Arsene, C, Braunschweig

Arslanoglu, J, New York, NY

Arvizu-Torres, MdR, El Marques

Arwin, H, Linköping

Ashby, J, Riverside, CA

Aspinwall, CA, Tucson, AZ

Aubriet, F, Metz
Augsburger, M, Lausanne

Avino, P, Rome

Ayesh, A, Al Ain

Ayora-Cañada, MJ, Jaén

Badea, S, Umeå

Baeumner, AJ, Regensburg

Bahrdt, C, Freiburg im Breisgau

Baker, J, Elmira, NY

Baker, MJ, Preston

Balagurunathan, K, Salt Lake City, UT

Balcaen, L, Ghent

Baldini, F, Sesto Fiorentino

Baldrich, E, Barcelona

Ballesteros, O, Granada

Bandowe, BAM, Bern

Banoub, J, St John's, NL

Bantscheff, M, Heidelberg

Bao, N, Nantong

Baptista, PV, Caparica

Baranov, V, San Fransico, CA

Barbas, C, Boadilla del Monte

Barding, G, Seattle, WA

Barron, LP, London

Barros, A, Aveiro

Barroso, M, Lisbon

Barsan, N, Tübingen 
Bartczak, D, Teddington

Barth, F, Columbus, $\mathrm{OH}$

Basile, F, Laramie, WY

Battu, S, Limoges

Baumgartner, S, Tulln an der Donau

Bazin, D, Paris

Bazylak, G, Bydgoszcz

Bean, HD, Hanover, NH

Bean, S, Manhattan, KS

Bearden, D, Charleston, SC

Beattie, JR, Dublin

Beck, A, St Julien en Genevois

Becker, M, Bremen

Beckmann, M, Aberystwyth

Behrenbruch, CP, Los Angeles, CA

Belder, D, Leipzig

Belkin, S, Jerusalem

Bell, S, Belfast

Bencsath, FA, Dauphin Island, AL

Bendle, J, Birmingham

Bengang, $X$, Singapore

Ben-Gigery, B, Vigo

Bengtson, A, Stockholm

Beni, S, Budapest

Beni, V, Linköping

Benkendorff, K, Lismore, NSW

Benter, T, Wuppertal

Berben, G, Gembloux

Beretta, G, Milan

Berezin, M, St Louis, MO

Berger, S, Leipzig

Berm, EJJ, Groningen

Bermejo, AM, Santiago de Compostela

Bermejo Barrera, P, Santiago de

Compostela

Berthiller, F, Tulln an der Donau

Berthod, A, Villeurbanne

Bertol, E, Florence

Bertoncello, P, Swansea

Bertucci, C, Bologna

Besler, M, Grosshansdorf

Bester, K, Essen

Bettmer, J, Oviedo

Beyene, NW, Addis Ababa

Bhargava, R, Urbana-Champaign, IL

Bicchi, C, Turin

Bilitewski, U, Braunschweig

Bings, NH, Hamburg

Birch, B, Luton

Birkemeyer, C, Leipzig

Birolo, L, Naples

Biziuk, M, Gdańsk

Black, R, Salisbury
Blanco-Gonzalez, E, Oviedo

Bleher, O, Tübingen

Blum, LJ, Villeurbanne

Blum, M-M, Munich

Blyth, A, Perth, WA

Bodemer, W, Göttingen

Bodoki, E, Cluj-Napoca

Boenke, A, Brussels

Boggs, A, Silver Spring, MD

Bon, D, Poitiers

Bonaccorsi, IL, Messina

Bondarenko, O, Tallinn

Bonifacio, A, Trieste

Bonn, GK, Innsbruck

Bordel, N, Oviedo

Borsdorf, H, Leipzig

Bortolotti, F, Verona

Bose, S, New York, NY

Bossee, A, Vert Le Petit

Bota, S, Oradea

Botha, A, Pretoria

Botre, F, Rome

Bottaro, CS, St John's, NL

Boulyga, C, Vienna

Boumba, VA, Ioannina

Bovee, TFH, Wageningen

Bowen, B, Berkeley, CA

Brandt, S, Liverpool

Brandtner, M, Vienna

Bredehoeft, M, Weiden i.d. OPf.

Bremser, W, Berlin

Brennan, J, Hamilton, ON

Brenton, G, Swansea

Brett, CMA, Coimbra

Britz-McKibbin, P, Hamilton, ON

Brock, R, Nijmegen

Broekaert, JAC, Hamburg

Brokl, M, Liege

Bromme, D, Vancouver, BC

Broothaerts, W, Ispra

Brown, RJC, Teddington

Brown, SD, Newark, NJ

Brüll, R, Darmstadt

Bryant-Friedrich, A, Toledo, $\mathrm{OH}$

Buchberger, W, Linz

Buckley, B, Piscataway, NJ

Bulska, E, Warsaw

Bunk, DM, Gaithersburg, MD

Burhenne, J, Heidelberg

Burke, D, West Lindfield, NSW

Busch, U, Oberschleissheim

Buszewski, B, Torun

Buttinger, G, Geel
Byrne, HJ, Dublin

Bystrom, C, Cleveland, $\mathrm{OH}$

Cabooter, D, Leuven

Cabovska, B, Milford, MA

Cai, Y, University Park, PA

Caixach, J, Barcelona

Cajka, T, Prague

Callebaut, AP, Tervuren

Calvete, J, Valencia

Cámara, C, Madrid

Camara, JE, Gaithersburg, MD

Câmara, JS, Funchal

Camoes, MF, Lisbon

Campíns-Falcó, P, Burjassot

Canals, A, Alicante

Canamares, MV, Madrid

Canário, J, Lisbon

Canela-Garayoa, R, Lleida

Cao, R, Chapel Hill, NC

Cao, Z, Jefferson, AK

Capriotti, Al, Rome

Carabias Martínez, R, Salamanca

Carda Broch, S, Castellón

Cárdenas Aranzana, S, Córdoba

Cardon, D, Lyon

Careri, M, Parma

Caretti, F, Rome

Carini, M, Milan

Carro, AM, Santiago de Compostela

Carvalho, PN, Aarhus

Castellino, S, Research Triangle Park, $\mathrm{NC}$

Castignolles, P, Penrith South DC, NSW

Castro, AL, Porto

Cataldi, TRI, Bari

Cavalca, V, Milan

Cavaliere, C, Rome

Cela Torrijos, R, Santiago de Compostela

Cevenini, L, Bologna

Chai, W, London

Challa, BR, Andhra Pradesh

Chan, ECY, Singapore

Chang, CA, Hsinchu

Chaniotakis, N, Iraklion

Chao, T-C, Regina, SK

Chaurand, P, Montreal, QC

Chen, C, Beijing

Chen, C-S, Jhongli

Chen, C-Y, Taipei City

Chen, F, Beijing

Chen, $\mathrm{H}$, Athens, $\mathrm{OH}$

Chen, K, Shreveport, LA 
Chen, L, Yantai

Chen, O, Boston, MA

Chen, P-K, Newark, NJ

Chen, T, Gainesville, FL

Chen, Y-L, Marlborough, MA

Chernetsova, ES, Moscow

Chiavarini, S, Rome

Chiba, H, Sapporo

Chilmonczyk, Z, Warsaw

Chisvert, A, Burjassot

Chiu, T-C, Taitung

Choi, HJ, Incheon

Christesen, S, Aberdeen Proving Ground, MD

Chung, SWC, Hong Kong

Cinta Pinzaru, S, Cluj-Napoca

Cioffi, N, Bari

Citterio, D, Yokohama

Clarke, M, Beltsville, MD

Clench, MR, Sheffield

Coffinier, Y, Villeneuve d'Ascq

Collins, CH, Campinas

Collins, M, Sydney, NSW

Colon, L, Buffalo, NY

Concheiro, M, Baltimore, MD

Conlan, XA, Geelong, VIC

Connelly, JT, Ithaca, NY

Cooks, RG, West Lafayette, IN

Cooper, A, Valhalla, NY

Corbisier, P, Geel

Corda, E, Madrid

Correia, M, Søborg

Costa-Fernández, JM, Oviedo

Costa-García, A, Oviedo

Couderc, F, Toulouse

Cox, HD, Salt Lake City, UT

Cox, JE, Salt Lake City, UT

Coym, JW, Mobile, AL

Cozzolino, D, Adelaide, SA

Craig, D, Winnipeg, MB

Crimmins, BS, Potsdam, NY

Cuadros-Rodríguez, L, Granada

Cui, G, Scottsdale, AZ

Cui, H, Hefei

Cui, S, Milwaukee, WI

Cui, W, St Louis, MO

Culbertson, CT, Manhattan, KS

Culha, M, Istanbul

Cunha, S, Porto

Cvacka, J, Prague

Dabrio Ramos, M, Geel

Dacres, H, Canberra, ACT
Dagnac, T, Abegondo

Dahlin, AB, Gothenburg

Dai, N, Ipswich, MA

Dall'Asta, C, Parma

Damian, G, Cluj-Napoca

Dang, F, Takamatsu

Danku, JMC, Aberdeen

Darie, CC, Potsdam, NY

Dauphin, Y, Orsay

Davies, N, Hobart, TAS

Davies, SR, Sydney, NSW

Davis, C, Charleston, SC

Dayon, L, Lausanne

De Brabanter, N, Jette

de Gennaro, G, Bari

De Jesus, VR, Atlanta, GA

de Jong, GJ, Utrecht

de la Fuente, JM, Zaragoza

de la Guardia, M, Burjassot

de la Torre, R, Barcelona

de la Torre, X, Rome

de Loos-Vollebregt, MTC, Delft

De Lorenzi, E, Pavia

de Mol, N, Utrecht

de Rijk, TC, Wageningen

De Saeger, S, Ghent

de Villiers, A, Matieland

de Vos, J, Pretoria

DeAngelis, PL, Oklahoma City, OK

Debrauwer, L, Toulouse

DeCaprio, AP, Miami, FL

Deckert, V, Jena

Deconinck, E, Brussels

Degano, I, Pisa

DeGroot, W, Freeport, TX

Degueldre, C, Villigen

Del Bubba, M, Sesto Fiorentino

Delay, M, Karlsruhe

Demeke, T, Winnipeg, MB

den Burger, JCG, Amsterdam

Denifl, S, Innsbruck

Denoroy, L, Lyon

Deprez, L, Geel

Desaire, H, Lawrence, KS

Desauziers, V, Pau

Desiderio, C, Rome

Desrosiers, NA, Baltimore, MD

Desta, Z, Indianapolis, IN

Dettmer, K, Regensburg

Devakumar, A, South San Francisco, CA

Deventer, K, Zwijnaarde

Di Carlo, D, Los Angeles, CA di Sabatino, M, Trondheim

di Targiani, R, Aberdeen Proving Ground, MD

Dibya, D, Ames, IA

Dickert, FL, Vienna

Dietliker, K, Zürich

Dietzek, B, Jena

Ding, Y, Gainesville, FL

Dixon, R, Lincoln

Doble, P, Broadway, NSW

Dolezal, K, Olomouc

Doménech-Carbo, M-T, Valencia

Dondi, F, Ferrarra

Dong, L, Beijing

D’Orazio, G, Monterotondo

Doucette, G, Charleston, SC

Dovichi, NJ, Notre Dame, IN

Drese, KS, Mainz

Drewnick, F, Mainz

Drews, O, Heidelberg

Drożdżyński, D, Poznań

Dubey, DK, Gwalior

Ducic, T, Cerdanyola del Vallès

Duewer, D, Gaithersburg, MD

Dufva, M, Kongens Lyngby

D’Ulivo, A, Pisa

Dunn, D, Pennington, NJ

Dwivedi, HP, Hazelwood, MO

Dzido, TH, Lublin

Ebrahimi, H, Edmonton, AB

Ehni, M, Dortmund

Einax, JW, Jena

Ekroos, K, Espoo

Elez-Martinez, P, Lleida

Ellisor, MB, Charleston, SC

Emons, H, Geel

Emslie, KR, Pymble, NSW

Emteborg, H, Geel

Engelhard, C, Siegen

Engewald, W, Leipzig

Enthaler, B, Hamburg

Erard, M, Orsay

Escarpa, A, Alcalá de Henares

Esposito, S, Zwijnaarde

Esseiva, P, Lausanne

Esteve-Romero, J, La Plana

Esteves da Silva, JCG, Porto

Evans-Nguyen, KM, Tampa, FL

Evtugyn, G, Kazan

Ewald, M, Tübingen

Ewing, AG, Gothenburg

Eyers, CE, Liverpool 
Fabbris, L, Piscataway, NJ

Fakhrullin, RFU, Kazan

Fanali, S, Monterotondo Scalo

Fantoni, R, Frascati

Favretto, D, Padova

Fearn, T, London

Fechner, P, Tübingen

Fedorov, E, Laval, QC

Fedorova, M, Leipzig

Fekete, J, Budapest

Feng, L, Appleton, WI

Feng, W, Beijing

Fernandez, JA, Leioa

Fernandez Abedul, MT, Oviedo

Fernández-Garcia, B, Oviedo

Fernandez-Piñas, F, Madrid

Fernández-Romero, JM, Córdoba

Fernandez-Sanchez, ML, Oviedo

Ferreira, CR, West Lafayette, IN

Ferrer, I, Boulder, CO

Fetzer, JC, Pinole, CA

Figueiredo, EC, Alfenas

Finney, L, Lemont, IL

Fisicaro, P, Paris

Flechsig, G-U, Rostock

Fleischer, M, Munich

Fletcher, JS, Gothenburg

Flora, JW, Richmond, VA

Flores, EMdM, Santa Maria

Fornal, E, Lublin

Fountain, AW, Aberdeen Proving Ground, MD

Foy, CA, Teddington

Francis, PS, Geelong, VIC

Franco, R, Caparica

Franke, AA, Honolulu, HI

Franzke, J, Dortmund

Freudenberger, K, Tübingen

Friedbacher, G, Vienna

Frolov, A, Leipzig

Frömel, T, Idstein

Fromell, K, Uppsala

Furey, A, Cork

Gaborieau, M, Penrith, NSW

Gagne, F, Montreal, QC

Gago-Martinez, A, Vigo

Gailer, J, Calgary, AB

Galceran Huguet, MT, Barcelona

Gallardo, E, Covilhã

Gamez, G, Lubbock, TX

Gammelgaard, B, Copenhagen

Ganeev, AA, St Petersburg
Gänzle, MG, Edmonton, AB

Garcia Alonso, JI, Oviedo

García Álvarez-Coque, MC, Burjassot

García-Barrera, T, Huelva

Gardinali, P, North Miami, FL

Gardner, P, Manchester

Garrett, TJ, Gainesville, FL

Garrido, M, Tarragona

Gaspar, EMMSM, Caparica

Gasparri, F, Nerviano

Ge, Y, Cranfield

Gediya, LK, Philadelphia, PA

Geissler, M, Hennef

Gensch, T, Jülich

Gerecke, AC, Dübendorf

Gervais, C, Bern

Gevaert, K, Ghent

Giersch, T, Menangle, NSW

Gil, R, San Luis

Gillespie, E, Sligo

Gilljam, BH, Kjeller

Glatz, Z, Brno

Godugu, B, Pittsburgh, PA

Goenaga-Infante, $\mathrm{H}$, Teddington

Goessler, W, Graz

Gomara, B, Madrid

Gomez, M, Madrid

Gomez Ariza, JL, Huelva

Gómez Caravaca, AM, Granada

Gómez Hens, A, Córdoba

Goncalves, C, Geel

González-García, MB, Porto

Goodpaster, JV, Indianapolis, IN

Gorecki, T, Waterloo, ON

Gorinstein, S, Jersualem

Gorris, H-H, Regensburg

Goryacheva, IY, Saratov

Gosetti, F, Alessandria

Gossett, DR, Los Angeles, CA

Graham, S, Belfast

Grassl, B, Pau

Grasso, G, Catania

Gray, SL, Clemson, SC

Grechnikov, A, Moscow

Gregg, H, The Hauge

Greiner, L, Mannheim

Griffiths, PR, Moscow, ID

Griffiths, W, Swansea

Grigorenko, AN, Manchester

Grob, K, Zürich

Groessl, M, Vienna

Gronwald, W, Regensburg

Groppi, A, Pavia
Gross, JH, Heidelberg

Grotemeyer, J, Kiel

Guan, F, West Chester, PA

Guardigli, M, Bologna

Guerrini, M, Milan

Guillarme, D, Geneva

Guillot, P, Albi

Günther, D, Zürich

Guo, M, Alhambra, CA

Guo, X, Cincinnati, OH

Guo, Y, Shanghai

Guo, Y, Teaneck, NJ

Gutiérrez Gallego, R, Barcelona

Gutleb, A, Belvaux

Hackley, VA, Gaithersburg, MD

Haertlé, T, Nantes

Hage, D, Lincoln, NE

Haglund, $\mathrm{P}$, Umeå

Hahn, O, Berlin

Hakkarainen, M, Stockholm

Halket, JM, London

Halsall, C, Lancaster

Hamase, K, Fukuoka

Hamm, G, Loos

Han, D, Hillsboro, OR

Hanai, T, Yokohama

Hann, S, Vienna

Hanrahan, G, Thousand Oaks, CA

Hao, Z, Piscataway, NJ

Haro, I, Barcelona

Harrison, CR, San Diego, CA

Harrison, WW, Gainesville, FL

Hartonen, K, Helsinki

Harynuk, JJ, Edmonton, AB

Hasegawa, T, Uji

Hattendorf, B, Zürich

Hauser, PC, Basel

Hayen, $\mathrm{H}$, Münster

Heal, M, Edinburgh

Heberger, K, Budapest

Heck, A, Utrecht

Hedegaard, M, Odense M

Heien, ML, Tucson, AZ

Heinisch, S, Villeurbanne

Heise, HM, Iserlohn

Heiskanen, A, Kongens Lyngby

Heitmann, Urs, Berlin

Henrion, A, Braunschweig

Hepel, M, Potsdam, NY

Heras, A, Burgos

Herckes, P, Tempe, AZ

Hercules, DM, Nashville, TN 
Hernández, F, Castellón

Hernández Redondo, A, Bern

Herrero, JM, Burjassot

Herrero, M, Madrid

Hess, P, Nantes

Hianik, T, Bratislava

Hibbert, DB, Sydney, NSW

Hieftje, GM, Bloomington, IN

Hietschold, M, Chemnitz

Hilbig, U, Tübingen

Hildebrandt, N, Orsay

Hilder, E, Hobart, TAS

Hill, HH, Pullman, WA

Himmelsbach, M, Linz

Hitzmann, B, Hannover

Ho, ENM, Hong Kong

Hoffmann, D, Burgos

Hoffmann, P, Adelaide, SA

Hoffmann, T, Mainz

Hoffmann, V, Dresden

Hofmann, T, Freising-Weihenstephan

Hola, K, Olomouc

Holčapek, M, Pardubice

Holland, LA, Morgantown, WV

Hollender, J, Dübendorf

Holley, J, Porton Down

Homola, J, Prague

Horváth, V, Budapest

Hosoya, K, Kyoto

Hou, X, Chengdu

Hou, X, New York, NY

Houk, RS, Ames, IA

Howes, M, London

Hsieh, Y, Kenilworth, NJ

Hsu, FF, St Louis, MO

$\mathrm{Hu}, \mathrm{B}$, Wuhan

$\mathrm{Hu}, \mathrm{W}$, Philadelphia, PA

$\mathrm{Hu}, \mathrm{Z}-\mathrm{Y}, \mathrm{Memphis,} \mathrm{TN}$

Huang, C-C, Guishan

Huang, HY, Zhongli

Huang, Q, Chongqing

Huang, T, Chapel Hill, NC

Huang, X, Beijing

Huck, C, Innsbruck

Hudson, E, Montreal, QC

Huestis, MA, Baltimore, MD

Huhn, C, Tübingen

Huijbrechts, AML, Utrecht

Hungerford, J, Bothell, WA

Hwang, G-S, Seoul

Ibanez, A, Zürich

Ifa, DR, West Lafayette, IN
Ikegami, T, Kyoto

Ilag, TL, Stockholm

Inagaki, K, Tsukuba

Infante, C, São Paulo

Ingendoh, A, Bremen

Iqbal, N, Islamabad

Irwin, PL, Wyndmoor, PA

Ishihara, K, Tokyo

Isobe, T, Matsuyama

Jaeger, S, Dessau-Rosslau

Jahouh, F, New York, NY

Jaisson, S, Reims

Jamting, ÅK, Lindfield, NSW

Jandera, P, Pardubice

Janfelt, C, Copenhagen

Janssen, H-G, Vlaardingen

Jarosz, M, Warsaw

Jeannot, R, Orleans

Jedziniak, P, Pulawy

Jenkins, GR, Kansas City, MO

Jensen, BP, Christchurch

Jensen, H, Copenhagen

Jeong, J-S, Daejeon

Ji, Y, Nanjing

Jia, M, Tucson, AZ

Jiang, J, Minneapolis, MN

Jiang, S, Lanzhou

Jiang, X, Chengdu

Jiang, X, San Jose, CA

Jin, F, Kannapolis, NC

Jin, W, Beijing

John, H, Munich

Johnson, R, Atlanta, GA

Josephs, RD, Sevres

Josic, D, Providence, RI

Julian, R, Riverside, CA

Juskowiak, B, Poznań

Jussi, M, Turku

Justino, C, Aveiro

Kaji, H, Sendai

Kakabakos, SE, Athens

Kaliszan, R, Gdańsk

Kalkum, M, Duarte, CA

Kamnev, AA, Saratov

Kannan, K, Albany, NY

Kanngießer, B, Berlin

Kara, P, Izmir

Karadag, R, Istanbul

Karapanagiotis, I, Thessaloniki

Karlovsky, P, Göttingen

Kataoka, H, Okayama
Katz, E, Potsdam, NY

Kaur, N, Chandigarh

Kazarian, SG, London

$\mathrm{Ke}, \mathrm{S}$, Chicago, IL

Keifer, PA, Mountain View, CA

Keire, DA, St Louis, MO

Kellner, R, Martinsried

Kempson, IM, Adelaide, SA

Kennedy, RT, Ann Arbor, MI

Kerman, K, Toronto, ON

Kessler, W, Reutlingen

Ketola, RA, Helsinki

Khan, I, Aveiro

Khoo, K-H, Taipei City

Kiefer, W, Würzburg

Kilpatrick, E, Gaithersburg, MD

Kilpatrick, L, Gaithersburg, MD

Kilz, P, Mainz

Kim, B, Yusong

Kim, K-H, Seoul

Kim, SS, Incheon

Kingston, HMS, Pittsburgh, PA

Kintz, P, Oberhausbergen

Kintzios, S, Athens

Kinumi, T, Tsukuba

Kissinger, PT, West Lafayette, IN

Kitagawa, S, Nagoya

Kitamori, T, Tokyo

Klaessig, FC, Doylestown, PA

Klavins, M, Riga

Klein, T, Landsberg am Lech

Klejdus, B, Brno

Knopp, D, Munich

Kobatake, E, Tokyo

Koch, BP, Bremerhaven

Koch, M, Berlin

Kolarov, F, Tübingen

Köllensperger, G, Vienna

Konarski, P, Warsaw

Konings, E, Lausanne

Kool, J, Amsterdam

Köper, I, Adelaide, SA

Köppen, R, Berlin

Kos, G, Montreal, QC

Koskela, H, Helsinki

Köszegi, T, Pecs

Kovačič, S, Ljubljana

Krachler, M, Heidelberg

Kraemer, T, Homburg (Saar)

Krafft, C, Jena

Krähenbühl, S, Basel

Kranz, C, Ulm

Krauss, M, Leipzig 
Kricka, LJ, Philadelphia, PA

Krishnan, S, Stillwater, OK

Kronstrand, R, Linköping

Krska, R, Tulln an der Donau

Krueger, T, Jena

Krüger, R, Karlsruhe

Krull, IS, Boston, MA

Krull, UJ, Mississauga, ON

Krystek, P, Eindhoven

Krzyzanek, V, Brno

Kubo, T, Kyoto

Kubota, LT, Campinas

Kuck, D, Bielefeld

Kuesters, M, Koblenz

Kuklenyik, Z, Atlanta, GA

Kumar, JR, Daejeon

Kumar, M, Patiala

Kuselman, I, Jerusalem

Kuster, B, Freising-Weihenstephan

Kylin, H, Linköping

Labat, L, Paris

Laborda, F, Zaragoza

Labuda, J, Bratislava

Lachman, J, Prague

Lacorte, S, Barcelona

Laemmerhofer, M, Tübingen

Laganà, A, Rome

Lagugné-Labarthet, F, London, ON

Lai, EPC, Ottawa, ON

Lai, Y, Chapel Hill, NC

Lakowicz, JR, Baltimore, MD

Lambropoulou, D, Thessaloniki

Lan, W, Cambridge, MA

Lanekoff, I, Richland, WA

Länge, K, Eggenstein-Leopoldshafen

Lao, W, Costa Mesa, CA

Laprevote, O, Paris

Larivière, D, Québec, QC

Larrechi, MS, Tarragona

Larsen, EH, Søborg

Larsen, M, Odense M

Larsen, R, St Mary's City, MD

Lattanzio, V, Bari

Lavine, BK, Stillwater, OK

Lavonen, E, Uppsala

Law, KP, Singapore

Le, XC, Edmonton, $\mathrm{AB}$

Le Blanc, AF, Munich

LeBeau, M, Quantico, VA

Lebedev, A, Moscow

Leblanc, RM, Coral Gables, FL

Lebrilla, C-H, Davis, CA
Leca-Bouvier, B, Lyon

Lechuga, LM, Tres Cantos

Lederer, A, Dresden

Lednev, IK, Albany, NY

Lee, DY, Berkeley, CA

Lee, HK, Singapore

Lee, S, Daegu

Lee, S, Taejon

Lee, YH, Daejeon

Lee, Y-M, Cheongju

Lehmann, WD, Heidelberg

Lehotay, SJ, Wyndmoor, PA

Lehr, M, Münster

Leis, HJ, Graz

Leitner, A, Zürich

Lemay, SG, Delft

Leng, G, Leverkusen

Leopold, K, Ulm

Leopold, N, Cluj-Napoca

Lerner, DA, Montpellier

Lesot, PGJ, Orsay

Letzel, T, Freising-Weihenstephan

Li, H, Beijing

Li, H, Santa Barbara, CA

Li, J, Beijing

Li, J, Charlottesville, VA

Li, K, East Lansing, MI

Li, L, Madison, WI

Li, N, Beijing

Li, QX, Honolulu, HI

Li, X, Athens, GA

Li, Z, Muncie, IN

Li, Z, Shanghai

Liao, Y, Wuhan

Lieberzeit, PA, Vienna

Lightfield, A, Wyndmoor, PA

Ligler, FS, Chapel Hill, NC

Lin, C-H, Taipei City

Lin, J, Waltham, MA

Lin, S, Hong Kong

Lindholm-Sethson, BD, Umeå

Lindner, W, Vienna

Lingeman, H, Amsterdam

Linhardt, RJ, Troy, NY

Linscheid, MW, Berlin

Linsinger, TPJ, Geel

Lintelmann, J, Neuherberg

Lippa, KA, Gaithersburg, MD

Liritzis, I, Rhodes

Lisdat, F, Wildau

Litescu, SC, Bucharest

Liu, H, Beijing

Liu, S, Beijing
Liu, W, Pasadena, CA

Liu, Y, Changchun

Liu, Y, Harrisonburg, VA

Liu, Z, Columbus, OH

Lloyd, GR, Gloucester

Lobo Castañón, M-J, Oviedo

Loeschner, K, Søborg

Loos, R, Ispra

López de Alda, M, Barcelona

Lorenzo, R, Santiago de Compsotela

Loroch, S, Dortmund

Lottspeich, F, Martinsried

Louchouarn, P, Galveston, TX

Lowe, AJ, Ithaca, NY

Lowenthal, MS, Gaithersburg, MD

Lu, C, Beijing

Lu, H, Nanjing

Lucena Rodríguez, R, Córdoba

Lundgren, E, Umeå

Lunte, S, Lawrence, KS

Lupoi, JS, Emeryville, CA

Luppa, P, Munich

Luque-Garcia, JL, Madrid

Lutter, $\mathrm{P}$, Lausanne

Lynen, F, Ghent

Ma, J, Baltimore, MD

Ma, M, Indianapolis, IN

Madariaga, JM, Leioa

Maher, W, Belconnen, ACT

Maksimov, I, Tsukuba

Malherbe, J, Gaithersburg, MD

Malhotra, BD, New Delhi

Malitesta, C, Lecce

Mallard, G, Gaithersburg, MD

Malmstadt, N, Los Angeles, CA

Mandler, D, Jerusalem

Manetta, AC, Teramo

Mangelings, D, Brussels

Mannelli, I, Castelldefels

Mano, N, Pessac

Maquieira, Á, Valencia

Marcelli, A, Frascati

Marchetti-Deschmann, M, Vienna

Marcus, RK, Clemson, SC

Markovic, G, Tübingen

Markuszewski, MJ, Gdańsk

Marques, JC, Funchal

Marquette, CA, Villeurbanne

Marshall, J, Toronto, ON

Martin, FL, Lancaster

Martín, MA, Madrid

Martin, RS, St Louis, MO 
Martinez Carballo, E, Ourense

Martínez-Chapa, SO, Monterrey

Martins, I, Alfenas

Märtlbauer, E, Munich

Mascolo, G, Bari

Maspoch, S, Bellaterra

Massolini, G, Pavia

Masson, JF, Montreal, QC

Matraszek-Zuchowska, I, Pulawy

Matulis, D, Vilnius

Maudens, KE, Wilrijk

Maurer, HH, Homburg (Saar)

Mayboroda, OA, Leiden

Mazak, K, Budapest

Mazzarino, M, Rome

McDonnell, L, Leiden

McGown, LB, Troy, NY

McLean, JA, Nashville, TN

McMahen, R, Durham, NC

Meagher, RJ, Livermore, CA

Medeiros, M, São Paulo

Meija, J, Ottawa, ON

Meisen, I, Münster

Melanson, JE, Halifax, NS

Menéndez-Carreño, M, Utrecht

Merkoci, A, Bellaterra

Merrick, BA, Research Triangle Park, $\mathrm{NC}$

Mester, Z, Ottawa, ON

Metsälä, M, Helsinki

Mezzanotte, L, Leiden

Michalke, B, Neuherberg

Michelini, E, Bologna

Michulec, MM, Lausanne

Miekisch, W, Rostock

Miliani, C, Perugia

Miller, JM, St Catharines, ON

Miller, M, Nelson

Minunni, M, Sesto Fiorentino

Mirasoli, M, Bologna

Mirkin, CA, Evanston, IL

Mirzaei, H, Dallas, TX

Mishra, YK, Kiel

Mitchell, J, Hamilton

Miyaguchi, H, Kashiwa

Mnatsakanyan, M, Sydney, NSW

Möder, M, Leipzig

Modesto, L, Kuopio

Mohs, AM, Winston-Salem, NC

Mol, HGJ, Wageningen

Moldovan, M, Oviedo

Molinié, R, Amiens

Mondello, L, Messina
Monis, P, Adelaide, SA

Montes-Bayón, M, Oviedo

Montoliu Roura, I, Lausanne

Montoya Baides, A, Valencia

Moon, MH, Seoul

Moore, DS, Los Alamos, NM

Moorthy, GS, Philadelphia, PA

Moradian, A, Pasadena, CA

Morais, S, Valencia

Morais, SB, Porto

Moreno Bondi, MC, Madrid

Morgan, SL, Columbia, SC

Morini, L, Pavia

Morlock, GE, Stuttgart

Mormann, M, Münster

Moscone, D, Rome

Moyano, E, Barcelona

Mroczek, T, Lublin

Muddiman, DC, Raleigh, NC

Mudiam, MR, Lucknow

Mueller, L, Berlin

Mueller, L, Riverside, CA

Muller, S, Strasbourg

Müller-Platz, C, Bonn

Mulloy, B, London

Mulvaney, S, Washington, DC

Munoz, A, Geel

Mura, U, Pisa

Muralidharan, S, Davis, CA

Murkovic, M, Graz

Murphy, KE, Gaithersburg, MD

Murray, J, Gaithersburg, MD

Musshoff, F, Bonn

Nadal, P, Tarragona

Nadeau, JL, Montreal, QC

Nakashima, K, Sasebo

Namiesnik, J, Gdańsk

Nanavati, D, Evanston, IL

Narizzano, R, Genoa

Natalini, B, Perugia

Natarajan, S, Beltsville, MD

Naughton, DP, London

Navas, N, Granada

Nebbioso, A, Portici

Nerin de la Puerta, C, Zaragoza

Neumann, S, Halle (Saale)

Neusüß, C, Aalen

Nevin, A, London

Newman, JW, Davis, CA

Nichols, KP, Lemont, IL

Nielen, M, Wageningen

Nielsen, KF, Ballerup
Niessen, W, Leiden

Niessner, R, Munich

Nilsson, L, Lund

Nogee, L, Baltimore, MD

Nogueira, ARdA, São Carlos

Nordkvist, E, Uppsala

Noser, J, Liestal

Nousiainen, M, Helsinki

Nugen, SR, Amherst, MA

Numata, M, Tsukuba

Oberacher, H, Innsbruck

O'Connor, G, Northwich

Oechsner, H, Kaiserslautern

Oeh, U, Munich

Oelkrug, D, Tübingen

Ogra, Y, Tokyo

Ohta, D, Sakai

Ojanperä, I, Helsinki

O'Keeffe, M, Dublin

Okino, A, Yokohama

Oliveri, P, Genoa

Olivieri, AC, Rosario

Olszowy, PP, Omaha, NE

Orellana, G, Madrid

Orlovius, A-K, Cologne

Östin, A, Umeå

O’Sullivan, CK, Tarragona

Ottens, AK, Richmond, VA

Oueslati, S, Borj-Cedria

Ozaki, Y, Sanda

Ozalp, VC, San Sebastian

Ozsoz, MS, Izmir

Öztürk, M, Muğla

Pabst, M, Zürich

Pacetti, D, Ancona

Pacheco, ME, La Plata

Paglia, G, Reykjavik

Pallaoro, A, Santa Barbara, CA

Palleschi, G, Rome

Palleschi, V, Pisa

Panderi, IE, Athens

Paolucci, F, Bologna

Pappas, D, Lubbock, TX

Parastar, H, Tehran

Park, S-R, Daejeon

Parshintsev, J, Helsinki

Pasch, H, Stellenbosch

Paschke, A, Hamburg

Pauwels, S, Leuven

Pawlak, K, Warsaw

Peel, S, Bristol 
Peeters, M, Diepenbeek

Pei, R, New York, NY

Pellati, F, Modena

Peltonen, K, Helsinki

Peng, Z, Boston, MA

Perdue, M, Muncie, IN

Pereiro, R, Oviedo

Perez Calderon, RA, Halifax, NS

Pérez-Pavón, JL, Salamanca

Pergantis, SA, Heraklion

Perr, J, Washington, DC

Perreau, F, Versailles

Pesavento, M, Pavia

Peters, FT, Jena

Petibois, C, Talence

Petr, J, Olomouc

Philipp, R, Berlin

Phillips, KS, Beltsville, MD

Phillips, MM, Gaithersburg, MD

Piccinelli, AL, Fisciano

Pichini, S, Rome

Pichon, V, Paris

Picó, Y, Burjassot

Pierce, K, Seattle, WA

Piergiovanni, AR, Bari

Piletska, E, Silsoe

Pingarrón, JM, Madrid

Pinheiro, T, Sacavem

Pino, V, La Laguna

Pisonero, J, Oviedo

Pitkänen, L, Gaithersburg, MD

Place, B, Gaithersburg, MD

Platz, S, Hamburg

Pleil, J, Chapel Hill, NC

Plumb, RS, London

Polesello, S, Brugherio

Poli, T, Turin

Poliwoda, AM, Opole

Poole, CF, Detroit, MI

Popp, J, Jena

Posthuma-Trumpie, GA, Wageningen

Prado, M, Braga

Pragst, F, Berlin

Prange, A, Geesthacht

Prashi, J, Lawrence, KS

Prati, S, Bologna

Pratt, KW, Gaithersburg, MD

Priego-Capote, F, Córdoba

Pritchard, C, London

Pritchett, J, Gaithersburg, MD

Pröfrock, D, Geesthacht

Prohaska, T, Tulln an der Donau

Proll, G, Tübingen
Provenzano, M, Zürich

Puettmann, W, Frankfurt am Main

Pumera, M, Singapore

Purves, RW, Saskatoon, SK

Pyell, U, Marburg

Qian, SY, Fargo, ND

Qiao, F, Baoding

Qiu, X, Princeton, NY

Quilliam, MA, Halifax, NS

Quintana, JB, Santiago de Compostela

Quintas, G, Valencia

Quirino, JP, Hobart, TAS

Raab, A, Aberdeen

Raba, J, San Luis

Rabbaa-Khabbaz, L, Beirut

Rabenstein, DL, Riverside, CA

Rabin, I, Berlin

Rader, JL, College Park, MD

Raimundo, IM, Campinas

Rambla-Alegre, M, Castello

Rampler, E, Vienna

Ranville, J, Golden, CO

Rastrelli, L, Salerno

Rathore, AS, New Delhi

Rau, S, Tübingen

Ravelo Pérez, LM, La Laguna

Rawn, DFK, Ottawa, ON

Ray, S, Bloomington, IN

Raymond, P, St Hyacinthe, QC

Read, R, Salisbury

Regal, P, Lugo

Rego, ECP, Duque de Caxias

Rehulka, P, Hradec Králové

Reid, GE, East Lansing, MI

Reinemann, C, Leipzig

Reiner, EJ, Toronto, ON

Reiner, JL, Gaithersburg, MD

Ren, J, Pittsburgh, PA

Rentsch, KM, Zürich

Resano, M, Zaragoza

Reschiglian, P, Bologna

Resmini, M, London

Reyzer, ML, Nashville, TN

Rhoderick, GC, Gaithersburg, MD

Ricci, M, Geel

Riches, J, Wiltshire

Richomme, P, Angers

Richter, E, Munich

Ridge, CD, College Park, MD

Riedel, J, Berlin

Righetti, PG, Milan
Rimmer, CA, Gaithersburg, MD

Rios-Castro, A, Ciudad Real

Ripp, S, Knoxville, TN

Rius Ferrus, FX, Tarragona

Rivera, J, Abbott Park, IL

Robb, P, Sand Hutton

Roberts, D, Liverpool

Roblyer, D, Boston, MA

Roda, B, Bologna

Rodda, LN, Southbank, VIC

Rodriguez-Gonzalez, P, Oviedo

Rodushkin, I, Luleå

Römpp, A, Giessen

Roper, MG, Tallahassee, FL

Rosenberg, E, Vienna

Rossini, GP, Modena

Roth, G, Freiburg im Breisgau

Ruan, C, Fayetteville, AR

Rubianes, MD, Córdoba

Rudaz, S, Geneva

Ruhlmann, L, Strasbourg

Ruiz Encinar, J, Oviedo

Rupp, F, Tübingen

Rybalchenko, I, Moscow

Rychlik, M, Freising-Weihenstephan

Ryzhov, V, DeKalb, IL

Sabbatini, L, Bari

Sabo-Attwood, T, Gainesville, FL

Sacher, F, Karlsruhe

Sagawa, T, Kyoto

Sahore, V, Provo, UT

Saito, K, Shinagawa-Ku

Saito, Y, Toyohashi

Saka, M, Jōsō

Salzer, R, Dresden

Samperi, R, Rome

Samyn, N, Brussels

Sanchez, JM, Girona

Sandau, CD, Calgary, AB

Sander, LC, Gaithersburg, MD

Santana Rodríguez, JJ, Las Palmas De G.C.

Santer, J, Auer

Santos, JL, Seville

Santos-Neto, AJ, São Carlos

Saraji, M, Isfahan

Sargent, M, Teddington

Satinsky, D, Hradec Králové

Sattler, W, Graz

Sauer, C, Jena

Scaffidi, JP, Durham, NC

Scalbert, A, Clermont-Ferrand 
Schantz, M, Gaithersburg, MD

Schaumlöffel, D, Pau

Scheller, FW, Potsdam-Golm

Scherer, G, Munich

Schiller, J, Leipzig

Schilling, K, Berkeley, CA

Schleicher, E, Tübingen

Schleifenbaum, F, Tübingen

Schlüter, H, Hamburg

Schmarr, H-G, Neustadt an der

Weinstraße

Schmidt, G, Aachen

Schmidt, TC, Essen

Schmitter, J-M, Bordeaux

Schmitz, OJ, Essen

Schneider, RJ, Berlin

Scholz, F, Greifswald

Scholz-Böttcher, B, Oldenburg

Schramm, K-W, Neuherberg

Schreiner, M, Vienna

Schubert, JK, Rostock

Schug, KA, Arlington, TX

Schuhmann, W, Bochum

Schwarz, B, Tübingen

Scriba, GKE, Jena

Seah, MP, Teddington

Seeber, R, Modena

Seidel, A, Grosshansdorf

Seidel, M, Munich

Sejeroe-Olsen, B, Geel

Sena, MM, Belo Horizonte

Serpe, M, Edmonton, AB

Sethi, M, Chapel Hill, NC

Setou, M, Fukushima

Seubert, A, Marburg

Sforza, S, Parma

Sgamellotti, A, Perugia

Shakleya, D, Baltimore, MD

Shanmugam, A, Valhalla, NY

Shard, A, Teddington

Shaw, PN, Brisbane, QLD

Shelley, J, Kent, OH

Shellie, RA, Hobart, TAS

Shen, F, Chicago, IL

Shen, S, Chapel Hill, NC

Shephard, GS, Tygerberg

Shi, X, Urbana-Champaign, IL

Shiea, J, Kaohsuing

Shieh, D-B, Tainan

Shimura, K, Fukushima

Shippy, S, Chicago, IL

Shore, LS, Bet Dagan

Shriver, Z, Cambridge, MA
Shundo, A, Fukuoka

Siano, S, Sesto Fiorentino

Silva, JAF, Campinas

Silva Lyra, W, João Pessoa

Simal-Gandara, J, Ourense

Simek, P, Ceske Budejovice

Simmons, BA, Livermore, CA

Simon, V, Toulouse

Simonian, A, Auburn, AL

Simonich, S, Corvallis, OR

Siripinyanond, A, Bangkok

Sitko, R, Katowice

Skladal, P, Brno

Skladanowski, AC, Gdańsk

Sklorz, M, Rostock

Skopp, G, Heidelberg

Skudra, A, Riga

Slade, D, Kansas City, MO

Sliwakowski, M, Warsaw

Sloth, JJ, Frederiksberg

Smadja, C, Chatenay Malabry

Smichowski, PN, San Martín

Smid, P, Marl

Smink, B, The Hauge

Smirnova, SV, Moscow

Smith-Palmer, T, Antigonish, NS

Snell, JP, Geel

Snow, NH, South Orange, NJ

Sobott, F, Antwerp

Söderberg, O, Uppsala

Soderstrom, M, Helsinki

Sojo, LE, Vancouver, BC

Solano, G, San Pedro

Song, D, Beijing

Song, NW, Daejeon

Soria, S, Sesto Fiorentino

Spence, DM, East Lansing, MI

Spinozzi, S, Bologna

Spitzer, P, Braunschweig

Spoto, G, Catania

Stadler, RH, Orbe

Stakenborg, T, Leuven

Stapleton, HM, Durham, NC

Stegemann, C, London

Steiner, G, Dresden

Stenken, JA, Fayetteville, AR

Stepan, R, Prague

Stigter, ECA, Utrecht

Stimpfl, T, Vienna

Stobiecka, M, Warsaw

Stoica, D, Paris

Stolk, LM, Maastricht

Stoll, D, St Peter, MN
Stout, P, Research Triangle Park, NC

Stove, CP, Ghent

Strano Rossi, S, Rome

Strehlitz, B, Leipzig

Striegel, A, Gaithersburg, MD

Stuart, JM, Warren, NJ

Stubiger, G, Vienna

Sturgeon, RE, Ottawa, ON

Stürup, S, Copenhagen

$\mathrm{Su}, \mathrm{Z}$, Waterloo, ON

Subramanian, S, Thanjavur

Suder, P, Krakow

Sugimoto, I, Tokyo

Sulé-Suso, J, Stoke on Trent

Sullards, MC, Atlanta, GA

Sulyok, M, Tulln an der Donau

Sun, W, Seattle, WA

Suzuki, S, Kashiwashi

Suzuki, T, Yokohama

Svec, F, Berkeley, CA

Sweedler, JV, Urbana-Champaign, IL

Synovec, R, Seattle, WA

Szabo, C, Deerfield, IL

Szökö, É, Budapest

Tabernero-Duque, MJ, Santiago de Compostela

Taitt, CR, Washington, DC

Takats, Z, Giessen

Takmakov, P, Silver Spring, MD

Taly, V, Paris

Tang, T, Kepala Batas

Tanner, SD, Toronto, ON

Tao, L, Boston, MA

Tarcomnicu, I, Bucharest

Tempez, A, Palaiseau

Teo, A, Singapore

Ternes, W, Hannover

Terry, P, Knoxville, TN

Teutenberg, T, Duisburg

Thakur, A, Rochester, NY

Thakur, MS, Mysore

Thelen, JJ, Columbia, MO

Thevis, M, Cologne

Thiébaut, D, Paris

Thiele, B, Jülich

Thiermann, H, Munich

Tholey, A, Kiel

Thomaidis, NS, Athens

Thompson, T, Edmonton, AB

Thorsén, G, Stockholm

Thouand, G, La Roche-sur-Yon 
Todoli, J-L, Alicante

Tombelli, S, Sesto Fiorentino

Tonoli, D, Geneva

Toth, I, Debrecen

Toyo'oka, T, Shizuoka

Traldi, P, Padova

Trapmann, S, Geel

Trimpin, S, Detroit, MI

Trtic-Petrovic, TM, Belgrade

Tsatsakis, AM, Heraklion

Tsim, KWK, Hong Kong

Tsochatzis, ED, Thessaloniki

Tsunoda, K-I, Kiryu

Tuoriniemi, J, Gothenburg

Turner, AD, Weymouth

Turrio-Baldassarri, L, Rome

Ueno, Y, Kanagawa

Ulberth, F, Geel

Unger, WES, Berlin

Urban, G, Freiburg im Breisgau

Vadgama, P, London

Vadillo, JM, Malaga

Van Berkel, GJ, Oak Ridge, TN

Van den Eede, G, Ispra

van Ginkel, L, Wageningen

Van Schepdael, A, Leuven

Vandenabeele, PJV, Ghent

Vanhaecke, F, Ghent

Vanhaecke, L, Merelbeke

Vashist, SK, Freiburg im Breisgau

Vautz, W, Dortmund

Venzago, C, Hanau

Verstraete, A, Ghent

Vertes, A, Washington, DC

Vickerman, J, Manchester

Vilaplana, F, Stockholm

Vilaró, F, Lleida

Villez, K, Dübendorf

Viñas López-Pelegrín, P, Murcia

Vincenti, M, Turin

Visconti, A, Bari

Vogel, M, Münster

Vogl, J, Berlin

Vogt, F, Knoxville, TN

Vogt, S, Argonne, IL

Völkel, W, Munich

Volmer, DA, Saarbrücken

von Holst, C, Geel

Voshol, J, Basel

Vovk, I, Ljubljana

Vuckovic, D, Montreal, QC
Vulliet, E, Villeurbanne

Vyskocil, L, Bratislava

Vyskocil, V, Prague

Wada, Y, Osaka

Wagatsuma, K, Sendai

Wagner, M, Indianapolis, IN

Wahlund, K-G, Lund

Walch, A, Munich

Walker, M, Teddington

Wallin, S, Tumba

Walorczyk, S, Poznań

Walsh, M, Urbana-Champaign, IL

Wang, C, North Miami, FL

Wang, E, Changchun

Wang, H, Palo Alto, CA

Wang, H-Y, Kaohsiung

Wang, J, La Jolla, CA

Wang, L, Hacienda Heights, CA

Wang, P-H, Beijing

Wang, Q, Xiamen

Wang, S, Baoding

Wang, T, Ann Arbor, MI

Wang, T, Petersburg, VA

Wang, W, Charlottesville, VA

Wang, W, Houston, TX

Wang, X, Suwanee, GA

Wang, Y, Atlanta, GA

Wang, Y, Boston, MA

Wang, Y, Minneapolis, MN

Wang, YK, Shanghai

Wang, Z, Beijing

Wang, Z, Seattle, WA

Warren, J, Teddington

Warth, B, Tulln an der Donau

Waters, JF, Gaithersburg, MD

Webb, MR, Wilmington, NC

Weber, G, Dortmund

Weber, P, Tübingen

Weber, SG, Pittsburgh, PA

Wegscheider, W, Leoben

Wei, F, Los Angeles, CA

Wei, G, Bremen

Wei, S, Richland, WA

Weimar, U, Tübingen

Weiner, B, Leipzig

Weinmann, W, Bern

Weitkamp, T, Gif-sur-Yvette

Welz, B, Santa Catarina

Wen, Y, Chicago, IL

Wenzel, TJ, Lewiston, ME

West, TP, Brookings, SD

Weusten, J, Geelen
White, E, Gaithersburg, MD

Whitesides, G, Cambridge, MA

Wickramasekara, S, Corvallis, OR

Widmaier, J, Tübingen

Wieczorek, PP, Opole

Wilkins, JA, Winnipeg, MB

Wille, SMR, Brussels

Williams, AM, Livermore, CA

Williams, G, Newport New, VA

Williams, K, Golden, CO

Williams, MTD, Pullman, WA

Williams, PJ, Pretoria

Willner, I, Jerusalem

Wilson, M, York

Winchester, MR, Gaithersburg, MD

Windust, A, Ottawa, ON

Winer, A, Los Angeles, CA

Wolf, WR, Beltsville, MD

Wolfbeis, OS, Regensburg

Wong, Y-C, Kowloon

Wong, Y-L, Kowloon Bay

Woolf, E, West Point, PA

Woolley, A, Provo, UT

Workman, W, Chesterfield, MO

Wozniak, B, Pulawy

Wrobel, K, Guanajuato

$\mathrm{Wu}, \mathrm{C}$, Acton, MA

Wu, M, Beijing

Wu, R, Atlanta, GA

$\mathrm{Wu}, \mathrm{S}$, Hangzhou

Wuhrer, M, Leiden

Xia, Y, West Lafayette, IN

Xiang, Y, Beijing

Xianghong, W, Baoding

Xiao, H, Los Angeles, CA

Xie, M-X, Beijing

Xie, Y, Macao

$\mathrm{Xu}, \mathrm{D}$, Nanjing

$\mathrm{Xu}, \mathrm{G}$, Dalian

$\mathrm{Xu}, \mathrm{G}$, Los Angeles, CA

$\mathrm{Xu}, \mathrm{W}, \mathrm{Mt}$ Pleasant, MI

$\mathrm{Xu}, \mathrm{Y}$, Manchester

Yakes, BJ, College Park, MD

Yamashita, K, Miyagi

Yan, F, Durham, NC

Yan, X, Xiamen

Yan, X-P, Tianjin

Yañez-Sedeño Orive, $\mathrm{P}$, Madrid

Yang, CJ, Xiamen

Yang, H(I), Piscataway, NJ 
Yang, L, Northfield, MN

Yang, L, Ottawa, ON

Yang, M, Atlanta, GA

Yang, PW, Etobicoke, ON

Yang, Y, Beijing

Yang, Y, Greenville, NC

Yang, Y-S, Hsinchu

Yantorno, OM, La Plata

Yao, X, Storrs, CT

Yarman, A, Potsdam-Golm

Yegles, M, Dudelange

Yeung, L, Toronto, ON

Yim, Y-H, Daejeon

Yin, J-H, Nanjing

Yonamine, M, São Paulo

YookHeng, L, Bangi

You, M, New York, NY

Yu, R, Chapel Hill, NC

$\mathrm{Yu}, \mathrm{X}(\mathrm{S})$, Evanston, IL

Yu, Y, Riverside, CA

Yu, Y, Rockville, MD

Yu, Z, Tempe, AZ

Yuan, M, Richmond, VA
Yuan, Y, Newark, DE

Yun, YH, Cincinnati, OH

Zaccheroni, N, Bologna

Zagatto, EAG, Piracicaba

Zaia, J, Boston, MA

Zanardi, C, Modena

Zangheri, M, Bologna

Zattoni, A, Bologna

Zavalin, A, Nashville, TN

Zaware, N, New York, NY

Zegers, I, Geel

Zeisler, R, Gaithersburg, MD

Zeleny, R, Geel

Zeng, Y, Lawrence, KS

Zenkevich, IG, St Petersburg

Zenobi, R, Zürich

Zhang, C, Riverside, CA

Zhang, H, Changchun

Zhang, L, Dalian

Zhang, Q, San Mateo, CA

Zhang, W, Boston, MA

Zhang, W, Milwaukee, WI
Zhang, X, Beijing

Zhang, X, Southampton

Zhang, Y, Cambridge, MA

Zhang, Y, Cincinnati, OH

Zhang, Y, Wuhu

Zhao, L, Mt Pleasant, MI

Zhao, Q, Taiyuan

Zhao, Y-D, Wuhan

Zheng, J, Silver Spring, MD

Zheng, M, Beijing

Zheng, P, Hefei

Zheng, W, Shanghai

Zhenli, Z, Wuhan

Zhong, W, Riverside, CA

Zhou, L, Chapel Hill, NC

Zhu, J, Ottawa, ON

Zhu, Z, La Jolla, CA

Zhu, Z, Seattle, WA

$\mathrm{Zi}, \mathrm{J}$, Ames, IA

Zimmermann, R, Rostock

Zong, Y, Singapore 